\title{
EFeito de Doses de Adubo 4-14-8 na CompetiçÃo ENTRE TOMATEIRO E Solanum americanum EM CONVIVÊNCIA INTRA E INTERESPECÍFICA ${ }^{1}$
}

\author{
Effect of Fertilizer 4-14-8 Doses on Competition Between Tomato and Solanum americanum \\ Under Intra- and Inter-Specific Coexistence
}

SILVA, B.P. ${ }^{2}$, CARVALHO, L.B. ${ }^{3}$ e ALVES, P.L.C.A. ${ }^{4}$

\begin{abstract}
RESUMO - O tomateiro (Lycopersicum esculentum) é uma das mais importantes hortaliças produzidas no mundo, porém sua produtividade pode ser reduzida em função da convivência com Solanum americanum (maria-pretinha). O objetivo desta pesquisa foi avaliar o efeito da adubação na relação de interferência intra e interespecífica entre plantas de tomateiro e S. americanum. Duas plantas em condições de convivência intra e interespecífica, por espécie, foram plantadas em vasos e adubadas com 13, 18 e $24 \mathrm{~g}$ de 4-14-8 por vaso, sendo avaliadas características de crescimento de ambas as espécies aos 90 dias após o transplante das plantas. A adubação com 4-14-8 estimulou o desenvolvimento da área foliar e da massa seca de caules, folhas e frutos de S. americanum, além da área foliar e da massa seca de folhas e frutos do tomateiro. A convivência interespecífica proporcionou maior altura de plantas de S. americanum, bem como menor altura e massa seca de folhas e frutos do tomateiro. Houve interação dos fatores adubação e convivência somente para o tomateiro, sendo a altura e a massa seca de folhas da cultura influenciadas negativamente quando submetidas às maiores doses de adubo e à competição com S. americanum.
\end{abstract}

Palavras-chave: adubação, Lycopersicum esculentum, maria-pretinha, interferência.

\begin{abstract}
Tomato (Lycopersicum esculentum) is one of the leading vegetable crops grown worldwide, but its productivity may be reduced due to coexistence with Solanum americanum (black nightshade). This work aimed to evaluate the effect of fertilization on intra-and inter-specific interference between tomato and $\mathbf{S}$. americanum plants. Two plants in intra-and inter-specific coexistence conditions of both species were planted in pots and fertilized with 13, 18 and $24 \mathrm{~g}$ of 414-8 per pot to evaluate the growth characteristics of both species at 90 days after transplanting. The 4-14-8 fertilization stimulated the development of the leaf area and dry mass of stems, leaves and fruit of S. americanum, consequently and equally influencing the leaf area and leaf and fruit dry mass of tomato. The inter-specific coexistence allowed a greater height of $\mathbf{S}$. americanum plants, promoting a lower height and dry mass of leaves and fruit of the tomato plants. There was interaction between the factors fertilization and coexistence only for the tomato plant. Crop height and leaf dry mass were affected negatively by $\mathbf{S}$. americanum competition and application of higher fertilizer rates.
\end{abstract}

Keywords: fertilization, Lycopersicum esculentum, American black nightshade, interference.

\section{INTRODUÇÃO}

O tomateiro (Lycopersicum esculentum) é uma das mais importantes hortaliças produzidas no mundo (Santos, 2009). O Brasil destaca-se como um dos maiores produtores dessa hortaliça, considerando a produção destinada à indústria, com área cultivada de aproximadamente 55.000 ha e uma produção de cerca de 3.200.000 toneladas, atingindo a

1 Recebido para publicação em 23.6.2009 e na forma revisada em 12.3.2010.

2 Engo-Agr - ${ }^{-}$, Dep. de Biologia Aplicada à Agronomia, Faculdade de Ciências Agrárias e Veterinárias, Universidade Estadual de São Paulo - FCAV/UNESP, 14884-900 Jaboticabal-SP, ${ }^{3}$ Bolsista CNPq, Doutorando em Produção Vegetal, FCAV/UNESP; ${ }^{4}$ Professor Livre Docente do Dep. de Biologia Aplicada à Agropecuária, FCAV/UNESP, Bolsista PQ-CNPq, <plalves@fcav.unesp.br>. 
produtividade de $58.000 \mathrm{~kg}^{\mathrm{g}}{ }^{-1}$ no ano de 2007 (Agrianual, 2008). Entretanto, a cultura do tomateiro está sujeita à interferência de plantas daninhas, que competem por nutrientes, água e luz e podem causar sérios prejuízos à cultura, reduzindo assim sua produtividade (Nascente et al., 2004; Hillger et al., 2006; Campiglia et al., 2009).

No tomateiro, plantas da família Solanaceae tornam-se muito importantes por pertencerem à mesma família botânica da cultura, o que dificulta o controle por meio de herbicidas. Duas espécies de plantas daninhas importantes no exterior são Solanum nigrum e Solanum ptycanthum, que foram relatadas causando perdas significativas na produtividade do tomateiro (McGiffen et al., 1994; Buckelew et al., 2006; Buckelew \& Monks, 2006). No Brasil, no caso do tomate rasteiro destinado prioritariamente à indústria, Solanum americanum destaca-se como uma planta daninha altamente prejudicial à cultura (Hernandez et al., 2007), por ser mais agressiva que o tomateiro quando em competição (Hernandez et al., 2002).

Hernandez et al. (2002) observaram que plantas de $S$. americanum acumulam mais massa seca e desenvolvem maior área foliar quando mantidas em convivência com o tomateiro, reduzindo, por consequência, tanto a massa seca acumulada como a área foliar da cultura. Hernandez et al. (2007) também verificaram reduções de produtividade maiores de $90 \%$ quando a convivência de $S$. americanum ocorre por todo o ciclo do tomateiro, observando, inclusive, aumento na incidência de podridão dos frutos; contudo, a cultura pôde conviver por 27 dias após o transplante com essa planta daninha sem que sua produtividade comercial fosse significativamente reduzida.

A competição das plantas daninhas com a cultura do tomateiro por nutrientes torna a adubação importante fator, que afeta a relação de interferência entre elas. Entretanto, não foi encontrada qualquer informação a respeito da resposta do tomateiro à adubação de plantio em competição com plantas daninhas. Assim, o objetivo desta pesquisa foi avaliar o efeito de doses do adubo 4-14-8 na relação de interferência intra e interespecífica entre plantas de tomateiro e $S$. americanum, baseando-se nas características de crescimento altura de plantas, área foliar e acúmulo de massa seca.

\section{MATERIAL E MÉTODOS}

O experimento foi realizado em vasos mantidos em ambiente aberto no Departamento de Biologia Aplicada à Agropecuária da Faculdade de Ciências Agrárias e Veterinárias, Universidade Estadual Paulista, campus de Jaboticabal-SP (FCAV/UNESP). Os vasos tinham capacidade para 10 litros e área de $0,05 \mathrm{~m}^{2}$, sendo preenchidos com terra proveniente da camada arável de um Latossolo Vermelho eutrófico típico. As características químicas do substrato eram: $\mathrm{pH}\left(\mathrm{CaCl}_{2}\right)$ de 5,9 ; $14 \mathrm{~g} \mathrm{dm}^{-3}$ de MO; $32 \mathrm{mg} \mathrm{dm}^{-3}$ de P; 2,4, 28, 13, 13, 43,4 e 56,4 mmol $_{\text {. }} \mathrm{dm}^{-3}$ de $\mathrm{K}, \mathrm{Ca}, \mathrm{Mg}, \mathrm{H}+\mathrm{Al}$, SB e T, respectivamente; e V de $77 \%$.

Não foi aplicado calcário. No plantio, aplicou-se a formulação de adubo 4-14-8 em três doses: $13 \mathrm{~g}$ por vaso (350 $\left.\mathrm{kg} \mathrm{ha}^{-1}\right), 18 \mathrm{~g}$ por vaso (500 $\left.\mathrm{kg} \mathrm{ha}^{-1}\right)$ e $24 \mathrm{~g}$ por vaso $\left(650 \mathrm{~kg} \mathrm{ha}^{-1}\right)$.

As mudas de tomateiro híbrido HEINZ 9992 e $S$. americanum foram transplantadas para os vasos 43 dias após a semeadura em bandejas de poliestireno expandido de 128 células preenchidas com substrato hortícola (Plantmax). $\mathrm{O}$ plantio das mudas foi feito nas seguintes condições de convivência intraespecífica: duas mudas de tomateiro por vaso e duas mudas de $S$. americanum por vaso; e convivência interespecífica: uma muda de tomateiro e uma muda de $S$. americanum por vaso.

Para cada espécie, os tratamentos foram dispostos em esquema fatorial $3 \times 2$, sendo as três condições de adubação como os fatores principais e as duas condições de convivência (inter e intraespecífica) como os fatores secundários, utilizando-se do delineamento experimental em blocos casualizados com quatro repetições.

Agrotóxicos foram aplicados semanalmente, a fim de garantir a sanidade das plantas de tomateiro e de $S$. americanum desde o estádio de muda até o final da fase experimental.

A avaliação de altura de plantas e área foliar e a colheita dos frutos foram realizadas 90 dias após o plantio. A altura de plantas foi medida utilizando uma régua graduada, 
mensurando-se a distância da base da planta até o último nó. A área foliar foi medida utilizando o aparelho Li-Cor LI3000A. Nessa ocasião, ainda, as plantas de ambas as espécies também foram coletadas, separadas nas diferentes partes (caules, folhas e frutos), lavadas e postas a secar em estufa de circulação forçada de ar a $70{ }^{\circ} \mathrm{C}$ por 96 horas. Posteriormente, o material seco foi pesado em balança de precisão de $0,01 \mathrm{~g}$, para estimativa do acúmulo de massa seca.

Os dados de altura, área foliar e massa seca das diferentes partes das plantas foram submetidos à análise de variância pelo teste $\mathrm{F}$, sendo as médias comparadas pelo teste de Tukey a $5 \%$ de probabilidade.

\section{RESULTADOS E DISCUSSÃO}

\section{Maria-pretinha}

A altura de plantas de $S$. americanum não foi afetada pela aplicação das diferentes doses do adubo 4-14-8, porém, quando a planta daninha foi mantida em convivência com a cultura, houve aumento na altura de plantas de S. americanum (Tabela 1), mostrando que

Tabela 1 - Efeito das doses do adubo 4-14-8 na altura, área foliar (AF) e massa seca de caule, folhas e frutos de Solanum americanum mantido em convivência com tomateiro. Jaboticabal-SP, 2008

\begin{tabular}{|c|c|c|c|c|c|}
\hline \multirow{2}{*}{ Fator } & \multirow{2}{*}{$\begin{array}{c}\text { Altura } \\
(\mathrm{cm})\end{array}$} & \multirow{2}{*}{$\begin{array}{c}\mathrm{AF} \\
\left(\mathrm{cm}^{2}\right)\end{array}$} & \multicolumn{3}{|c|}{ Massa seca (g) } \\
\hline & & & Caules & Folhas & Frutos \\
\hline \multicolumn{6}{|c|}{ Doses (D) } \\
\hline $\mathrm{D}_{1}$ & $49,67 \mathrm{~A}$ & $665,71 \mathrm{~B}$ & $5,73 \mathrm{~B}$ & $6,53 \mathrm{~B}$ & $10,83 \mathrm{~B}$ \\
\hline $\mathrm{D}_{2}$ & $50,58 \mathrm{~A}$ & $809,73 \mathrm{~B}$ & $6,60 \mathrm{~B}$ & $8,62 \mathrm{AB}$ & $15,51 \mathrm{AB}$ \\
\hline $\mathrm{D}_{3}$ & $50,50 \mathrm{~A}$ & $1.247,06 \mathrm{~A}$ & $8,90 \mathrm{~A}$ & $10,13 \mathrm{~A}$ & $20,42 \mathrm{~A}$ \\
\hline \multicolumn{6}{|c|}{ Convivência (C) } \\
\hline $\mathrm{C}_{\text {inter }}$ & $55,89 \mathrm{~A}$ & $998,54 \mathrm{~A}$ & $6,97 \mathrm{~A}$ & $8,77 \mathrm{~A}$ & $13,39 \mathrm{~A}$ \\
\hline $\mathrm{C}_{\text {intra }}$ & $44,61 \mathrm{~B}$ & $816,46 \mathrm{~A}$ & $7,18 \mathrm{~A}$ & $8,10 \mathrm{~A}$ & $17,79 \mathrm{~A}$ \\
\hline $\mathrm{F}_{\mathrm{D}}$ & $0,26^{\mathrm{NS}}$ & $9,7 * *$ & $8,93 * *$ & $4,07 *$ & $6,24 *$ \\
\hline $\mathrm{F}_{\mathrm{C}}$ & $95,83 * *$ & $2,63^{\mathrm{NS}}$ & $0,11^{\mathrm{NS}}$ & $0,43^{\mathrm{NS}}$ & $3,94^{\mathrm{NS}}$ \\
\hline$F_{\text {DXC }}$ & $0,04^{\mathrm{NS}}$ & $2,11^{\mathrm{NS}}$ & $0,53^{\mathrm{NS}}$ & $0,40^{\mathrm{NS}}$ & $2,00^{\mathrm{NS}}$ \\
\hline $\mathrm{DMS}_{\mathrm{D}}$ & 3,76 & 366,47 & 2,07 & 3,40 & 7,24 \\
\hline $\mathrm{DMS}_{\mathrm{C}}$ & 2,51 & 244,46 & 1,38 & 2,26 & 4,83 \\
\hline $\mathrm{CV}(\%)$ & 4,86 & 26,24 & 19,05 & 26,1 & 30,16 \\
\hline
\end{tabular}

$\mathrm{D}_{1}=13 \mathrm{~g}, \mathrm{D}_{2}=18 \mathrm{~g}, \mathrm{D}_{3}=24 \mathrm{~g}$ da formulação $4-14-8$ por vaso. $\mathrm{C}_{\text {inter }}-$ convivência interespecífica, $\mathrm{C}_{\text {intra }}-$ convivência intraespecífica. ${ }^{\text {NS }}$ não significativo; $*$, $*$ significativo a 5 e $1 \%$ de probabilidade pelo teste $\mathrm{F}$, respectivamente. Médias seguidas por mesma letra na coluna não diferem entre si pelo teste de Tukey a $5 \%$ de probabilidade. houve estimulo de crescimento em altura da planta daninha pela convivência com a cultura. A área foliar e a massa seca de caules de plantas de $S$. americanum foram maiores quando se aplicaram $24 \mathrm{~g}$ por vaso do adubo, porém, quando a planta daninha foi mantida em convivência com a cultura, não houve influência na área foliar e na massa seca de caules das plantas de $S$. americanum (Tabela 1), mostrando que o uso da maior dose de adubo 4-14-8 promoveu maior desenvolvimento da área foliar e acúmulo de massa seca nos caules da planta daninha. As massas secas de folhas e frutos de plantas de S. americanum foram afetadas pela aplicação das diferentes doses do adubo, e houve diferença apenas entre a dose de $13 \mathrm{~g}$ por vaso e a de $24 \mathrm{~g}$ por vaso; todavia, quando a planta daninha foi mantida em convivência com a cultura, não houve influência na massa seca de folhas e frutos das plantas de S. americanum (Tabela 1), mostrando que o uso de maiores doses do adubo 4-14-8 promoveu maior acúmulo de massa seca por folhas e frutos de $S$. americanum.

Por ser mais agressiva que o tomateiro (Hernandez et al., 2002), S. americanum cresceu mais em altura quando mantida a competição interespecífica, buscando estabelecer-se acima do dossel da cultura para intensificar a absorção de luz e melhorar, assim, o desempenho fotossintético. Além disso, evidencia-se que o acúmulo de massa seca de $S$. americanum não foi afetado negativamente pela convivência com o tomateiro (Tabela 1), mostrando que a competição interespecífica não influenciou o desenvolvimento da planta daninha. Ressalta-se que, em determinadas situações, maiores quantidades de nutrientes podem ser aproveitadas pelas plantas daninhas quando se aplicam doses maiores de fertilizantes (Tomaso, 1995; Patterson, 1995; Procópio et al., 2005), o que explica o fato de a planta daninha, no geral, ter se desenvolvido mais com a aplicação das maiores doses do adubo.

Não foi observada qualquer interação entre os fatores dose de adubo e condição de convivência para $S$. americanum (Tabela 1).

\section{Tomateiro}

A altura de plantas do tomateiro não foi afetada pela aplicação das diferentes doses do 
adubo, porém, quando a cultura foi mantida em convivência com a planta daninha, houve redução na altura de plantas do tomateiro (Tabela 2). Isso mostra que a competição interespecifica foi prejudicial à cultura em relação à altura das plantas. Contudo, a área foliar do tomateiro foi afetada pela aplicação das diferentes doses do adubo 4-14-8; houve diferença apenas entre as doses de $13 \mathrm{~g}$ por vaso e $18 \mathrm{~g}$ por vaso. Quando a cultura foi mantida em convivência com a planta daninha, não houve influência na área foliar das plantas do tomateiro (Tabela 2), mostrando que o uso adequado de adubo auxiliou no desenvolvimento da área foliar das plantas.

A massa seca de caules do tomateiro não foi afetada nem pela aplicação das diferentes doses do adubo nem pela convivência com a planta daninha (Tabela 2), mostrando que tanto as doses de 4-14-8 quanto a convivência intra e interespecífica não influenciaram o acúmulo de massa seca pelos caules do tomateiro. A massa seca de folhas do tomateiro foi afetada pela aplicação das diferentes doses do adubo, sendo maior quando se utilizou a dose de $18 \mathrm{~g}$ por vaso; além disso, quando a cultura foi mantida em convivência com a planta

Tabela 2 - Efeito das doses do adubo 4-14-8 na altura, área foliar $(\mathrm{AF})$ e massa seca de caule, folhas e frutos do tomateiro mantido em convivência com Solanum americanum. Jaboticabal-SP, 2008

\begin{tabular}{|l|c|c|c|c|c|}
\hline \multirow{2}{*}{ Fator } & Altura & \multirow{2}{*}{$\begin{array}{c}\text { AF } \\
(\mathrm{cm})\end{array}$} & \multicolumn{3}{|c|}{ Massa seca $(\mathrm{g})$} \\
\cline { 4 - 6 } & $\left(\mathrm{cm}^{2}\right)$ & Caules & Folhas & Frutos \\
\hline \multicolumn{6}{|c|}{ Doses $(\mathrm{D})$} \\
\hline $\mathrm{D}_{1}$ & $51,83 \mathrm{~A}$ & $1.148,36 \mathrm{~B}$ & $6,40 \mathrm{~A}$ & $14,09 \mathrm{~B}$ & $29,25 \mathrm{~B}$ \\
\hline $\mathrm{D}_{2}$ & $53,17 \mathrm{~A}$ & $1.779,50 \mathrm{~A}$ & $8,04 \mathrm{~A}$ & $18,10 \mathrm{~A}$ & $39,68 \mathrm{AB}$ \\
\hline $\mathrm{D}_{3}$ & $55,25 \mathrm{~A}$ & $1.510,83 \mathrm{AB}$ & $7,80 \mathrm{~A}$ & $10,81 \mathrm{~B}$ & $51,09 \mathrm{~A}$ \\
\hline \multicolumn{6}{|c|}{ Convivência $(\mathrm{C})$} \\
\hline $\mathrm{C}_{\text {inter }}$ & $50,67 \mathrm{~B}$ & $1.527,72 \mathrm{~A}$ & $7,27 \mathrm{~A}$ & $11,65 \mathrm{~B}$ & $26,79 \mathrm{~B}$ \\
\hline $\mathrm{C}_{\text {intra }}$ & $56,17 \mathrm{~A}$ & $1.431,44 \mathrm{~A}$ & $7,55 \mathrm{~A}$ & $17,02 \mathrm{~A}$ & $53,23 \mathrm{~A}$ \\
\hline $\mathrm{F}_{\mathrm{D}}$ & $1,66^{\mathrm{NS}}$ & $6,50^{*}$ & $2,29^{\mathrm{NS}}$ & $12,48^{* *}$ & $8,73^{* *}$ \\
\hline $\mathrm{F}_{\mathrm{C}}$ & $12,68^{* *}$ & $0,45^{\mathrm{NS}}$ & $0,17^{\mathrm{NS}}$ & $20,19^{* *}$ & $38,36^{* *}$ \\
\hline $\mathrm{F}_{\mathrm{DXC}}$ & $5,52 *$ & $3,70^{\mathrm{NS}}$ & $0,69^{\mathrm{NS}}$ & $8,07^{* *}$ & $1,43^{\mathrm{NS}}$ \\
\hline $\mathrm{DMS}_{\mathrm{D}}$ & 5,04 & 468,29 & 2,21 & 3,89 & 13,94 \\
\hline $\mathrm{DMS}_{\mathrm{C}}$ & 3,36 & 312,38 & 1,50 & 2,59 & 9,29 \\
\hline $\mathrm{CV}(\%)$ & 6,13 & 20,56 & 19,33 & 17,66 & 22,63 \\
\hline
\end{tabular}

$\mathrm{D}_{1}=13 \mathrm{~g}, \mathrm{D}_{2}=18 \mathrm{~g}, \mathrm{D}_{3}=24 \mathrm{~g}$ da formulação $4-14-8$ por vaso. $\mathrm{C}_{\text {inter }}$ - convivência interespecífica, $\mathrm{C}_{\text {intra }}$ - convivência intraespecífica. Ns não significativo; ${ }^{*}, * *$ significativo a 5 e $1 \%$ de probabilidade pelo teste $\mathrm{F}$, respectivamente. Médias seguidas por mesma letra na coluna não diferem entre si pelo teste de Tukey a $5 \%$ de probabilidade daninha, houve redução na massa seca de folhas de plantas do tomateiro (Tabela 2), o que indica tanto que o uso adequado de adubo auxiliou no acúmulo de massa seca de folhas das plantas, quanto que a competição interespecífica foi prejudicial à cultura. A massa seca de frutos do tomateiro foi afetada pela aplicação de diferentes doses do adubo; houve diferença apenas entre a dose de $13 \mathrm{~g}$ por vaso e a de $24 \mathrm{~g}$ por vaso. Quando a cultura foi mantida em convivência com a planta daninha, houve redução na massa seca de frutos de plantas do tomateiro (Tabela 2), mostrando tanto que a adubação auxiliou no acúmulo de massa seca de frutos, quanto que a competição interespecífica foi prejudicial à cultura.

Hernandez et al. (2002) afirmaram que a área foliar é a característica de crescimento mais sensivel à competição interespecífica entre o tomateiro e $S$. americanum. Relataram também que plantas dessas espécies não competem pelos mesmos recursos do meio em relação ao acúmulo de massa seca. Contudo, neste trabalho, observou-se que a área foliar do tomateiro não foi alterada pela interferência de $S$. americanum e que, em contrapartida, a massa seca de folhas e frutos foi reduzida, não corroborando, portanto, os resultados obtidos pelos referidos pesquisadores. Provavelmente, a divergência entre esses resultados pode ter ocorrido em função da época de coleta das plantas para avaliação. Hernandez et al. (2002) avaliaram o experimento aos 30 dias após o transplante, ao passo que este experimento foi avaliado no estádio produtivo da cultura. No período em que os referidos autores realizaram o experimento, as plantas das duas espécies estavam em estádio inicial de desenvolvimento. Segundo Fayad et al. (2002) e Silva et al. (2003), nesse período, o crescimento e o requerimento nutricional do tomateiro são baixos. Isso possibilita à planta daninha, mais agressiva, alocar mais eficientemente recursos do meio para seu crescimento.

McGiffen et al. (1994) observaram que $S$. nigrum e $S$. ptycanthum reduziram o número de frutos por planta do tomateiro importante característica produtiva dessa cultura. Buckelew et al. (2006) verificaram que $S$. ptycanthum reduziu o peso e o valor comercial dos frutos do tomateiro. Buckelew \& Monks (2006) observaram redução no 
número e na massa fresca de frutos, ocasionando perdas substanciais de produtividade do tomateiro, quando submetido à interferência de S. ptycanthum. Por serem essas espécies semelhantes a $S$. americanum, evidencia-se que a planta daninha estudada pode reduzir significativamente a produtividade do tomateiro, caso seja mantida em convivência com a cultura.

Dos três macronutrientes fornecidos na adubação deste experimento, o potássio é acumulado em maior quantidade pelo tomateiro, seguido por nitrogênio e fósforo (Fayad et al., 2002). Ainda segundo esses pesquisadores, o maior acúmulo de macro e micronutrientes pelo tomateiro intensifica-se depois dos 30 dias após o transplante, atingindo o máximo na época da colheita. Do total de nitrogênio, fósforo e potássio acumulados pelo tomateiro na época da colheita, 55, 54 e 56\%, respectivamente, estavam alocados nos frutos (Fayad et al., 2002), evidenciando a importância da adubação N-P-K para essa cultura. Segundo Ferreira et al. (2006), a adubação nitrogenada influenciou diretamente o aumento do teor de clorofila em plantas de tomateiro, o que pode aumentar a taxa fotossintética e promover melhor desenvolvimento da cultura. A adubação fosfatada é de extrema importância para a cultura do tomateiro, pois proporciona aumento no peso médio dos frutos (Alvarenga, 2004). Ainda segundo esse último pesquisador, a adubação potássica pode aumentar em $30 \%$ a produtividade do tomateiro, impede a queda e melhora a qualidade comercial dos frutos.

Houve ainda interação entre os fatores dose aplicada do adubo e condição de convivência para as variáveis altura de plantas e massa seca de folhas do tomateiro (Tabela 2).

Quando houve convivência interespecífica, a altura de plantas do tomateiro não foi alterada pela aplicação de diferentes doses do adubo, porém, no caso da convivência intraespecífica, a altura de plantas do tomateiro foi maior nas doses de $24 \mathrm{~g}$ por vaso do adubo; além disso, a dose do adubo não influenciou a altura de plantas quando a convivência foi interespecífica, mas, na dose de $24 \mathrm{~g}$ por vaso do adubo, plantas de tomateiro ficaram mais altas quando mantidas em convivência intraespecífica (Tabela 3). Esses fatos evidenciam que o maior fornecimento de adubo possibilitou maior crescimento das plantas de tomate quando em convivência intraespecífica, ou seja, há melhor aproveitamento do adubo para o crescimento quando a planta de tomate possui, em sua vizinhança, outra planta de tomate.

Quando houve convivência interespecífica, a massa seca de folhas de plantas do tomateiro foi menor ao serem aplicados $24 \mathrm{~g}$ por vaso do adubo, o que não foi observado quando a convivência foi intraespecífica; contudo, nessa dose, plantas de tomateiro ficaram mais altas quando mantidas em convivência intraespecífica (Tabela 4). Esses fatos mostram que a planta daninha beneficiou-se mais da adubação em maior dose, demonstrando maior desvantagem do tomateiro na competição interespecífica, para esta característica. Isso ocorre em função da maior agressividade da planta daninha, como relatado por Hernandez et al. (2002).

Tabela 3 - Efeito da interação entre as doses do adubo 4-14-8 e as condições de convivência sobre a altura do tomateiro mantido em convivência com Solanum americanum. Jaboticabal-SP, 2008

\begin{tabular}{|c|c|c|}
\hline Fator & $\mathrm{C}_{\text {inter }}$ & $\mathrm{C}_{\text {intra }}$ \\
\hline $\mathrm{D}_{1}$ & $50,00 \mathrm{Aa}$ & $53,67 \mathrm{Ab}$ \\
\hline $\mathrm{D}_{2}$ & $53,00 \mathrm{Aa}$ & $53,33 \mathrm{Ab}$ \\
\hline $\mathrm{D}_{3}$ & $49,00 \mathrm{Ba}$ & $61,50 \mathrm{Aa}$ \\
\hline
\end{tabular}

$\mathrm{D}_{1}=13 \mathrm{~g}, \mathrm{D}_{2}=18 \mathrm{~g}, \mathrm{D}_{3}=24 \mathrm{~g}$ da formulação $4-14-8$ por vaso. $\mathrm{C}_{\text {inter }}-$ convivência interespecífica, $\mathrm{C}_{\text {intra }}-$ convivência intraespecífica.Médias seguidas por mesma letra não diferem entre si pelo teste de Tukey a $5 \%$ de probabilidade; letras maiúsculas comparam os efeitos de convivência dentro das doses (horizontal) e minúsculas, os efeitos de doses dentro de convivência (vertical).

Tabela 4 - Efeito da interação entre as doses do adubo 4-14-8 e as condições de convivência sobre a massa seca de folhas do tomateiro mantido em convivência com Solanum americanum. Jaboticabal-SP, 2008

\begin{tabular}{|c|c|c|}
\hline Fator & $\mathrm{C}_{\text {inter }}$ & $\mathrm{C}_{\text {intra }}$ \\
\hline $\mathrm{D}_{1}$ & $13,10 \mathrm{Aa}$ & $15,08 \mathrm{Aa}$ \\
\hline $\mathrm{D}_{2}$ & $17,12 \mathrm{Aa}$ & $19,08 \mathrm{Aa}$ \\
\hline $\mathrm{D}_{3}$ & $04,74 \mathrm{Bb}$ & $16,88 \mathrm{Aa}$ \\
\hline
\end{tabular}

$\mathrm{D}_{1}=13 \mathrm{~g}, \mathrm{D}_{2}=18 \mathrm{~g}, \mathrm{D}_{3}=24 \mathrm{~g}$ da formulação $4-14-8$ por vaso. $\mathrm{C}_{\text {inter }}$ - convivência interespecífica, $\mathrm{C}_{\text {intra }}-$ convivência intraespecífica.Médias seguidas por mesma letra não diferem entre si pelo teste de Tukey a $5 \%$ de probabilidade; letras maiúsculas comparam os efeitos de convivência dentro das doses (horizontal) e minúsculas, os efeitos de doses dentro de convivência (vertical). 
A cultura do tomateiro é altamente exigente em nutrientes, porém a absorção desses elementos essenciais é baixa até o início do florescimento (Silva et al., 2003). Logo, nessa fase inicial, $S$. americanum pode competir intensamente por esses recursos do solo, diminuindo sua disponibilidade para o tomateiro e reduzindo, assim, o desenvolvimento da cultura. Esse fator, aliado à competição por luz, pode explicar o menor desenvolvimento do tomateiro mantido em convivência com S. americanum.

Conclui-se que a convivência de plantas de $S$. americanum com plantas de tomateiro é prejudicial ao desenvolvimento da cultura mesmo quando se aumenta a dose aplicada do adubo 4-14-8.

\section{AGRADECIMENTOS}

Ao PIBIC/CNPq, pelo apoio e incentivo.

\section{LITERATURA CITADA}

AGRIANUAL: Anuário da agricultura brasileira. São Paulo: FNP Consultoria, 2008. p. 478-482.

ALVARENGA, M. A. R. Tomate: produção em campo, em casa-de-vegetação e em hidroponia. Lavras: Universidade Federal de Lavras, 2004. 400 p.

BUCKELEW, J. K. et al. Eastern black nightshade (Solanum ptycanthum) reproduction and interference in transplanted plasticulture tomato. Weed Sci., v. 54, n. 3, p. 490-495, 2006.

BUCKELEW, J. K.; MONKS, D. W. Effect of eastern black nightshade (Solanum ptycanthum) on transplanted plasticulture tomato grade and yield. Weed Sci., v. 54, n. 3, p. 504-508, 2006.

CAMPIGLIA, E. et al. The effects of cover cropping on yield and weed control of potato in a transitional system. Field Crops Res., v. 112, n. 1, p. 16-23, 2009.

FAYAD, J. A. et al. Absorção de nutrientes pelo tomateiro cultivado sob condições de campo e de ambiente protegido. Hortic. Bras., v. 20, n. 1, p. 90-94, 2002.
FERREIRA, M. M. M. et al. Índice Spad e teor de clorofila no limbo foliar do tomateiro em função de doses de nitrogênio e da adubação orgânica, em duas épocas de cultivo. R. Ceres, v. 53, n. 305, p. 83-92, 2006.

HERNANDEZ, D. D. et al. Períodos de interferência de maria-pretinha sobre tomateiro industrial. Hortic. Bras., v. 25 , n. 2 , p. $199-204,2007$.

HERNANDEZ, D. D.; ALVES, P. L. C. A.; SALGADO, T. P. Efeito da densidade e proporção de plantas de tomate industrial e de maria-pretinha em competição.

Planta Daninha, v. 20, n. 2, p. 229-236, 2002.

HILLGER, D. E. et al. Weed management systems in Indiana tomato production. Weed Sci., v. 54, n. 3, p. 516-520, 2006.

MCGIFFEN, M. E.; PANTONE, D. J.; MASIUNAS, J. B.

Path analysis of tomato yield, quality, and competition with black and eastern black nightshade (Solanum nigrum L. and S. ptycanthum Dun.). J. Am. Soc. Hortic. Sci., v. 119, n. 1, p. 6-11, 1994.

NASCENTE, A. S.; PEREIRA, W.; MEDEIROS, M. A. Interferência das plantas daninhas na cultura do tomate para processamento. Hortic. Bras., v. 22, n. 3, p. 602-606, 2004.

PATTERSON, D. T. Effects of environmental stress on weed/ crop interactions. Weed Sci., v. 43, n. 3, p. 483-490, 1995.

PROCÓPIO, S. O. et al. Absorção e utilização do fósforo pelas culturas da soja e do feijão e por plantas daninhas. R. Bras. Ci. Solo, v. 29, n. 6, p. 911-921, 2005.

SANTOS, B. M. Drip-applied metam potassium and herbicides as methyl bromide alternatives for Cyperus control in tomato. Crop Protec., v. 28, n. 1, p. 68-71, 2009.

SILVA, J. B. C. et al. Embrapa hortaliças. Cultivo de tomate para industrialização. Nutrição e adubação. 2003.

Disponível em: <http://

sistemasdeproducao.cnptia.embrapa.br/FontesHTML/

Tomate/TomateIndustrial/adubacao.htm $>$. Acesso em: 04 jun. 2009.

TOMASO, J. M. Approaches for improving crop competitiveness through the manipulation of fertilization strategies. Weed Sci., v. 43, n. 3, p. 491-497, 1995. 